\title{
Larva Migrans Visceral en Niños
}

\author{
Dra. Isabel Noemi H. ${ }^{1}$; Dra. Waitraud Schuh O. ${ }^{2}$; Dr. Pedro Herskovic L. ${ }^{3}$; Dr. Emesto Ríos L. ${ }^{2}$ \\ Sr. Luis Cerva C. ${ }^{1}$; Sra. María Teresa Torres $C^{1}$; Sr. Renzo Tassara $0 .{ }^{4}$; \\ Sra. M. Еlena Urzúa 4; Dra. Patricia dal Borgo A.2 ; Dra. Carmen Gutiérrez S. 5
}

\section{Visceral Larva Migrans}

Forty two children with clinical and serological evidence Visceral Larva Migrans Infection are presented. Their mean age was 4 years 2 months \pm 3 years 5 months, $97,6 \%$ of patients had pica, in $64,3 \%$ there was pulmonary involvement and in $59,5 \%$ hepatomegaly. Abnormalities of the eye fundus were found in 4,8\% of the children. Mean Hemoglobin level was $11,0 \mathrm{~g} / \mathrm{dl} \pm 1.4$, mean Leucocite count was $19.213 / \mathrm{mm}^{3}$, mean absolute count of eosinophils in blood was $8.918 .9 / \mathrm{mm}^{3} .84,8 \%$ of this children had anti $A$ or anti $B$ isoaglutins titers $\geqslant 1: 64$. ELISA titers for anti Toxocaral antibodies were $\geqslant 1: 64$ in $95,3 \%$ of the patients. Treatment with Tiabendazole was well tolerated in $83,3 \%$ of the children and was considered effective in $70,8 \%$

Se denomina síndrome de Larva migrans visceral a la entidad clínica producida en el hombre, secundaria a la migración sistemática de larvas đel género Toxacaral.

El agente más frecuente de este cuadro es Toxacara canis, cuyo huésped definitivo es el perro, pero también puede producirse secundariamente a la presencia en el organismo de Toxacara catis (gato) u otros 2 .

Diversos estudios señalan que la infección afecta entre 2 y $100 \%$ de la población canina en diferentes paises del mundo del mundo ${ }^{3}$. En Chile se ha comunicado infección en 23 y $40 \%$ de los perros de hasta un año de edad 4-5.

El hombre adquiere la infección al ingerir huevos de Toxocara sp desde la tierra contaminada por las deposiciones de cachorros infectados ${ }^{2}$.

1 Servicio de Parasitología, Hospital Luis Calvo Mackenna. Unidad de Parasitología, División Ciencias Médjcas Oriente Universidad de Chile.

2 Servicio de Hematología, Hospital Luis Caro Mackenna.

Institu to de Salud Pública.

4 Internos 70 Año de Medicina, Facultad de Medicina, Div. Ciencias Médicas Oriente, Universidad de Chile.

Servicio de Gastroenterología, Hospital Luis Calvo Mackenna.
Ciertamente por hábitos y actitudes, son los niños de corta edad, los más susceptibles a contraer la infección ${ }^{3}$. Una vez ingeridos los huevos salen de éstos larvas que atraviesan la pared intestinal, iniciando una migración sistémica, sin completar su ciclo de vida en el hombre, que produce granulomas altededor de las larvas del nemátodo, tratando de encapsularlo e inmovilizarlo. Todos los parénquirnas del cuerpo pueden ser afectados pero con mayor frecuencia se ha descrito el compromiso pulmonar y hepático ${ }^{6}$.

Las manifestaciones clinicas descritas por Beaver en 1952 varían, desde casos asintomáticos a otros severos con hepatomegalia, anemia, eosinofulia intensa, síntomas pulmonares recidivantes que se acompañan de antecedente de geofagia, concentraciones plasmáticas de isoaglutininas y ganaglobulinas elevadas ${ }^{7}$, lo que corresponde a la forma sistemática de la infección. Existen también modalidades clínicas localizadas como la forma ocular, en que el hallazgo principal es la larva con su granuloma en el ojo, sin las características clínicas descritas en los casos sistémicos 8. Al parecer existen además, entre ambas entida. des clínicas bien definidas, otras mixtas ${ }^{6}$. El curso hacia una $u$ otra modalidad parece depender de factores del huésped, como la calidad de su respuesta inmune $\mathrm{y}$ de factores del parásito 
.tales como su capacidad antigénica y cantidad ${ }^{6}$. Experimentalmente se ha comprobado que con la ingestión de 200 huevos larvados de Toxocara canis, se produce una enfermedad sistérnica cuya duración oscila entre 12 y 36 meses $^{3-9-27}$. En un estudio previo de nifros que presentaban eosinofilia en el área Oriente de Santiago, se comprobó que 4,4\% correspondian probablemente al síndrome de Lava migrans visceral ${ }^{10}$.

El propósito de esta comunicación es dar a conocer el estudio de 42 casos clínicos de síndrome de Larva migrans visceral, diagnosticados mediante la técrica ELISA*9.

\section{MATERIAL Y METODOS}

Los pacientes fueron $\mathbf{4 2}$ niños con eosinofilia sobre 500 células $\times \mathrm{mm}^{3}$ estudiados entre el segundo semestre de 1979 y el últímo de 1983 , 23 eran del sexo femenino.

Todos los niños fueron interrogados buscando antecedentes clinicos que orientasen a infeccio-" nes parasitarias, enfermedades alérgicas, gastrointestinales, tumorales y hereditarias que pudieran explicar el aumento de eosinófilos circulantes.

En cada uno se efectuó examen parasitológico seriado de deposiciones ( 3 muestras) según el método de Teleman modificado ${ }^{12} \mathrm{y}$ prueba de Graham (5 muestras) ${ }^{13}$.

Según los antecedentes clínicos se realizaron intradermorreacciones para Distoma, Cisticercosis, reacción de Bachman ${ }^{14}$; pruebas serologicas de Fijación del Complemento para Distoma y Cisticercosis ${ }^{15.17}$; Floculación con Bentonita y Precipitinas para triquinosis ${ }^{16-17}$; Inmunoelectroforesis para hidatidosis ${ }^{\mathbf{1}}$; Reacciones de Sabin y Feldman, Hemaglutinación y Fijación del Complemento para Toxoplasmosis 19-20 y además ELISA para Larva migrans (Center for Disease Control, Atlanta, Georgia, U.S.A.) considerando, por no haber experiencias nacionales, como positivos los títulos para Toxara en diluciones de $1: 32$, de acuerdo a la información publicada en los Estados Unidos ${ }^{1} 1$.

Una vez confirmado el diagnóstico del síndrome Larva migrans visceral, se hizo en todos los njưos titulación de isoglutininas anti $\mathrm{A} y$ anti $B^{21}$ y examen de fondo de ojos.

Los pacientes fueron tratados con Tiabenda-

\footnotetext{
* Nota del Editor.

ELISA es un ensayo de Inmunoabsorción en fase sólida de semejante diseño que un radio-inmuno ensaya, pero en lugar de utilizar un isótopo radioactivo como marcador de las inmuno gdobulinas emplea una enzima. La sigla corresponde a las expresiones del inglés Enzime-linked inmunosorbent assay.
}

zol $30 \mathrm{mg} \times \mathrm{kg} \mathrm{x}$ día por 5 días ${ }^{2-23}$ repitiéndose un mes después el mismo esquema, con controles mensuales de su evolución clínica y hematológica.

\section{RESULTADOS}

La edad promedio de los pacientes infectados por larvas de Toxocara sp fue de 4 años 2 meses \pm 3 años 5 meses con un rango de 1 año 7 meses a 14 años, su peso promedio era de $17,1 \pm 9,9 \mathrm{~kg}$ con valores extremos de 9,6 y $49 \mathrm{~kg}$ en el momen. to del estudio y el promedio de la talla $98,0 \pm 19,8$ $\mathrm{cm}$ con rangos de 71 a $148 \mathrm{cms}$.

En 41 casos $(97,6 \%)$ habia geofagia; contacto con perros en $76,2 \% ; 64,3 \%$ de los pacientes tenía antecedentes de tos, disnea y signos bronquiales obstructivos recidjvantes, que causaron numerosas consultas, pero sólo $28,6 \%$ presentó complicaciones como evidencia de condensaciones que, radiológicamente, correspondieron a infiltrados pulmonares cambiantes. Habia Hepatomegalia en 26 casos $(59,5 \%)$, con proyección hepática, promedio de $2 \mathrm{~cm} \pm 0,5 \mathrm{~cm}$ bajo el reborde costal, de consistencia normal. Se consta. tó anorexia en $45,2 \%$ de los niños, diarrea en $35,7 \%$; fiebre en $28,6 \%$, variando de 37,8 a $39^{\circ} \mathrm{C}$ cuando estaba presente.

Se observó palidez de piel y mucosas en 6 casos $(14,3 \%)$ y fondo de ojos alterado en dos niños que tenían formaciones quísticas intrapapilares con bandas vítreas (Tabla 1).

La concentración promedio de hemoglobina en la sangre fue de $11,0 \mathrm{~g} / \mathrm{dl} \pm 1,4 \mathrm{~g} / \mathrm{dl}, 33$ niños $(78,6 \%)$ tenían cifras menores, en ellos la anemia era de características hiporegenerativas.

El promedio de leucocitos $/ \mathrm{mm}^{3}$ fue de $19.213,2$ con rangos de 9.000 a 92.000 . El recuento porcentual promedio de eosinófilos fue $35,8 \%$ con rangos de 8 a $75 \%$ y su recuento absoluto de $8.918 / \mathrm{mm}^{3}\left(1.088\right.$ a $\left.69.000 / \mathrm{mm}^{3}\right)$.

En general la infección fue bien tolerada, habiéndose hospitalizado sólo aquellos niños que presentaron como complicación sindrome de condensación pulmonar que en algunos casos requirio, además del tratamiento antibiótico habitual, el uso de corticoides.

La prueba de ELISA para antígenos de Toxo* cara (Tabla 2) dio resultados que en la gran mayoría de los casos se concentraron alrededor de títulos de 1:256 $(61,9 \%)$. Sólo $4,8 \%$ de la muestra ( 2 niños) presentaron títulos en el límite inferior de 1:32.

En la mayor parte de los pacientes se encontró elevación de las isohemaglutininas. Un 60\% del total de los pacientes, tuvo reacciones positivas en diluciones superiores a 1:128 (Tabla 3). 
Tabla 1.

Antecedentes clínicos en 42 casos de Larva Migrans Visceral. Confimada serolögicamente por Elisa

Edad promedio:

$\pm \mathrm{SD}=4$ años 2 meses \pm 3 años 5 meses.

Peso promedio:

$\pm \mathrm{SD}=17.1 \mathrm{Kg} . \pm 9.9 \mathrm{Kg}$.

Talla promedio:

$\pm \mathrm{SD}=98.0 \mathrm{~cm} \pm 19.8 \mathrm{~cm}$.

\section{Antecedentes cliricos y síntoinas:}

$\begin{array}{llc} & \text { N } & \% \\ \text { Geofagia } & 41 & 97,6 \\ \text { Contacto con perros } & 32 & 76,2 \\ \text { Tos, disnea y desgarro } & 27 & 64,3 \\ \text { Contacto con gatos } & 23 & 54,8 \\ \text { Anorexia } & 19 & 45,2 \\ \text { Diarrea } & 15 & 35,7 \\ \text { Rash cutáneo máculopapuloso } & 13 & 30,9\end{array}$

Hallazgos al examen físico:

Hepatomegalia

Sindrome bronquial obstructivo

Síndrome de condensación

Fiebre (37.8 - 39)

Palidez de piel y mucosas

Esplenomegalia

Fondo de ojos alterado

Tabla 2.

Distribución de los Títulos de Elisa para Taxocara (CDC, Atlanta, Georgia)

\begin{tabular}{lrr}
\hline & \multicolumn{1}{c}{$\mathrm{n}=42$} \\
Título & \multicolumn{1}{c}{$\mathrm{N}$} & \\
\hline $1: 32$ & 2 & 4,8 \\
$1: 64$ & 8 & 19,0 \\
$1: 128$ & 6 & 14,3 \\
$\geqslant 1: 256$ & 26 & 61,9 \\
\hline
\end{tabular}

Tabla 3 .

Distribución de los Títulos de lsoaglutininas Anti A O Anti B

\begin{tabular}{lrc} 
& $\mathrm{n}=33$ \\
Título & $\mathrm{N}$ & $\%$ \\
\hline $1: 32$ & 5 & 15,2 \\
$1: 64$ & 8 & 24,2 \\
$1: 128$ & 6 & 18,2 \\
$\geqslant 1: 256$ & 14 & 42,4 \\
\hline
\end{tabular}

Rango $1: 32$ a $1: 4096$

Veinticuatro nifnos han completado el tratamiento con Tiabendazol, en $20(83,3 \%)$ fue bien tolerado, y dos sufrieron exantemas confluentes, fugaces que desaparecieron dos dias después de suspender la droga. Un niffo tuyo Hiperpirexia y otro náuseas y vómitos que desaparecieron al suspender el medicamento.

La droga dio buenos resultados en el 70,8\%:17 de los niños, normalizándose los eosinófilos entre 3 y 6 meses de control. En 7 casos sólo se observó reducción parcial de los síntomas con persistencia de la eosinofilia, aunque de menor magnitud que al inicio del estudio $(29,2 \%)$, debiendo repetir el tratamiento en 364 oportunidades, hasta obtener desaparición de los síntomas y normalización del recuento de eosinófilos y leycocitos circulantes.

\section{DISCUSION}

Existen pocas publicaciones nacionales sobre esta enfermedad en nifos ${ }^{24.25}$ y se refieren a casos aislados. Sin embargo en vista de esta serie se puede afimnar que la prevalencia de Toxocariasis es mayor que la aparente por las comunicaciones mencionadas. En ciertas regiones de Chile se dan las condiciones para que esta infección ocurra con más frecuencia que lo comunicado, como probablemente perros infectados sin controles adecuados, que diseminan huevos de Toxocara canis en la tierra ${ }^{4-5}$. Los huevos deben madurar en el ambiente para hacerse infectantes ${ }^{2} y$ sobre. viven en él varios meses ${ }^{26}$. La ingestión de tierra contaminada produce la infección humana ${ }^{6}$. La prevalencia de infección canina depende del grado de sanearniento ambiental, desarrollo socioeconómico, clima $\mathrm{y}$ humedad de los diferentes países $^{3-4-5}$. Es evidente que los niños, por sus hábitos son muy susceptibles a la infección ${ }^{24-}$ 25-27-28- 29 .

Los antecedentes cl il ... de esta serie coinciden con los de otros autores en las estrechas relaciones entre geofagia, infección y eosinofilia. El contacto estrecho con perros no guarda una correlación tan estrecha, si se guardan las medidas preventivas adecuadas ${ }^{6}$.

También es coincidente esta experiencia con la de otros autores ${ }^{2}$ en que los síntomas pulmonares recidivantes, cuya intensidad es variable constituyen una de las manifestaciones más frecuentes $(63 \%)$, cuya patogenia estaría determinada por la presencia de larva y producción de granulomas, que clínicamente se traducen en episodios bronquiales obstructivos, neumonitis o síndrome dd condensación de curso variable 1-3? 
Estos síntomas acompañados o no por hepato. megalia moderada en pacientes con leucocitosis, eosinofilia absoluta elevada deben hacer pensar en la toxocariasis en niños sin otras molestias importantes.

Puede haber anemia en estos casos, pero es de poca magnitud, bien tolerada e hiporegenerativa. $\mathrm{La}$ elevación de las isohemaglutininas ${ }^{30}$ observa. da en la mayoría de estos niffos, puede ayudar a orientar el diagnóstico ${ }^{2}$. Se desconoce el meca. nismo mediante el cual aumentan las isohemaglutininas anti $A$ o anti $B$, pero se postula que el parásito tiene determinantes antigénicos que provocan su elevación 6 .

El diagnóstico serológico mediante la reacción de ELISA tiene una especificidad de $76,9 \%$ y una sensibilidad de $91,3 \% 11$. Aunque en Chile no se han comunicado los títulos diagnósticos, en la mayor parte de estos casos, éstos se concentraron muy por encima de $1: 32$, considerado limite para el điagnóstico en U.S.A.

El diagnóstico por punción biopsia, aunque permita observar el parásito, se considera poco útil, por su bajo rendimiento pues se trata de un procedimiento a ciegas que no asegura la obtención de nematodos ${ }^{32}$.

El Tiabendazol es una droga cuyo efecto antihelmíntico ha sido comunicado en repetidas oportunidades 22-23. En general fue bien tolera. da. Los efectos adversos observados remitieron poco después de suspender el medicamento. La respuesta al tratamiento fue satisfaçtoria tanto clínica como hematológicamente y coincide con la experiencias comunicada por otros autores ${ }^{2-23}$. Los resultados no satisfactorios $(29,2 \%)$ coincidieron con nifios que siguieron comiendo tierra, por lo que es difícil saber si el tratamiento tue insuficiente o hubo reinfección por el parásito.

\section{RESUMEN}

Se estudiaron clinicamente y mediante exáme. nes de laboratorio 42 niños infectados por Toxocara sp. La edad promedio de los casos fue de 4 años 2 meses \pm 3 affos 5 meses. Había geofagia en $97,6 \%$, grados variables de compromiso pulmonar en $64,3 \%$ y hepatomegalia en $59,5 \%$ de los pacientes. El Fondo de ojos tenja alteraciones en 4,8\% de los pacientes. En el Hemograma la concentración de Hemoglobina promedio fue de $11,0 \mathrm{~g} / \mathrm{dl}$, el recuento de leucocitos promedio $19.213 \times \mathrm{mm}^{3}$ y el de eosinófilos $8.918,9 \times$ $\mathrm{mm}^{3} .84,86 \%$ de los nifios presentaron niveles de isohemaglutininas $\geqslant 1,64$ y $95,2 \%$ títulos de ELISA para Toxocara superiores a esta dilución.
El tratamiento con Tiabendazol fue bien tolerado en $83,3 \%$ y efectivo en $70,8 \%$ de los niños. En $29,2 \%$ se observó mejoría parcial, clínica y de laboratorio.

\section{AGRADECIMIENTOS}

A "Parasitology Division of the Center for Disease Control, Atlanta, Georgia", por la realización del diagnóstico serológico de Toxocariasis.

Al personal del Banco de Sangre del Hospital Luis Calvo Mackenna Sras. María Agurto, Mónica Arizmendi, Irene Ruiz y Sr. Miguel Manosalva, por la titulación de jsohemaglutininas.

\section{REFERENCIAS}

${ }^{1}$ Huntley C.C., Costas M.C., Lyerly A.: Visceral larva migrans syndrome: clinical characteristics and immunological in 51 patients. Pediatr. 36: 523, 1965.

${ }^{2}$ M. Fanning M.D. A. Hill M.D., H.M. Langer M.D., J.S. Keystone M.D.: Visceral Jarva migrans (toxocariasis) in Toronto. Can. Med. Assoc. J. 124: 21 , 1981.

3 A. Burke Joseph M.D.: Visceral larva migrans (Toxocariasis). J.Ky. Med. Assoc. 75: 67, 1977.

4 Alcaino A., Tagle J.: Estudio de en teroparasitosis del perro en Santiago. Bol. Chile Parasit. 25: 5, 1970.

s oberg C., Franjola K., Leyan V.: Helmintos del perto doméstico, canis familiaris en la ciudad de Valdivia. Bol. Chile Parasit. 34: $21,1979$.

- Schaniz M. Peter, Glickman T. Lawrence.: Toxocara visceral larva migrans. N. Engl. J. Med. 298: 436, 1978.

${ }^{7}$ Beaver P.C., Synder C.H., Carrera G.M., et al.: Chronic eosinophilia due to visceral larva migrans: report of three cases. Pediatrics. 9: 7, 1952.

${ }^{8}$ Schantz Peter M., Meyer David and Glickman Lawrence,: Clinical serologic and epidemiologic characteristics of ocular Toxocariasis. An. J. Trop. Med. Hyg. 28: 24, 1979.

9 Mok C.H.: Visceral larva migrans: a discussion based on review of the literature. Clin. Pediatr. 7: 565, 1968.

10 Noemi I., Schuh W.: Etiología Parasitaria de las eosinofilias en la infancia. Parasit. al día IV: 87 , 1980.

11 Glickman L., Schantz P., Dombroske R., Cypess P.: Evaluation of serodiagnostic tests for visceral larva migrans. An. J. Trop. Med. Hyg. 27; 492, 1978.

12 Sapero J.J., Lowless D.K. and Stine L.D.: An impro. ved iodine staining for routine laboratory diagnosis of intestinal protozoa, Science 114: $550,1951$.

13 A device for the diagnosis of $E$. vermicularis infection, Am. J. Trop. Med. 31: 159, 1941.

14 Knierem $F_{*}:$ El valor de las reacciones serológicas en el diagnóstico de algunas infecciones parasitarias. Bol. Chile Parasit. 19: 119, 1964.

15 Barriga O.O.: Bases moleculares de las reacciones inmunológicas en infecciones parasjtarias. Bol. Chile Parasitol. 27: 42, 1972.

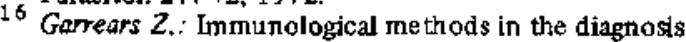
of husnan and animal trichinosis. Exp. Med. Microbjol. $20: 219,1963$. 
${ }^{7}$ Kegan I.G.: Advances in the Immunodiagnosis of Parasitic infections. Parasitenk 45: 163, 1974.

18 Capron $A$., Vernes $A$, et Bigue? $C$ : Le diagnostic immunoelectroforetique del hydatidose en le kyste hydatique du foil. Yournets Lyonnaises de Hydatido logie. SIMEP. Ed. Lyon pag. 27-40, 1967.

19 Thierman E., Knierem F. and Niedman G.: Vergleichende untersuchen über die Sabin-Feldman reaktionen und den Haema aglutinations tests bei In fectionen mit Toxoplasma gondii. Zbl Bakt 192: 230, 1964.

${ }^{20}$ Knierim $F,:$ 1958. Técnica de reacción de fijación del complemento según el métado del $50 \%$ de hemólisis de Bozicevich oplicada al diagnóstico de enfermedad de Chagas. Bol. Chile Parasit. 13: 75, 1958.

21 Isohemagiutins. Blaod Transfusion.: P.L. Mollison. p. 409. Blackwell ScientificsPublications Oxford and Edinburg, 1967.

22 Rhomes I.A., Aur M.D., Chates B. Pratt M.D., Warren W. Johnson M.D., Memphis.: Tiabendazole in Visceral larva migrans Amer. J. Dis. Child. 12 L: 226, 1971.

23 William C. Campbelt, PHD.: Thiabendazole effects in
Visceral larva migrans. J.A.M.A. 217: 342, 1971.

24 Leiva $H_{\text {, }}$ León F., Romero P., Salgado $C_{1}$, Benavente S.: Larva migrans visceralis. Rev. Chil. Pediat. 50: $53,1979$.

25 Aldunate $D_{.}$, Pérez $C_{\text {, }}$ Hidalgo $C_{\text {.: }}$ Larva migrans visceral. Rev. CChil. Pediat. 54: 258, 1983.

26 Cypess R.H.: Visceral larva migrans. Comell Vet. 68, 283. 1978.

27 Smith M.H.D., Beqver P.C.: Persistence and distribution of Toxocas a larvas in the tissues of children and mice. Pediatt. 12: 491, 1953.

28 Coldwell J., Labell M., Coceto P.: Mitogenic response to Toxocara antigen and chemotatic defect in visceral larra migrans. J. Dis. Child 134: 845, 1980.

29 Hetko P.. Patel Ch.; Sealer R.: Visceral larva mi grans. Ill. Med. J. 144: $574,1973$.

30 Huntley G., Ryerly A., Patterson M.: Isohemagglutinits in parasitic infections. J.A.M.A. 208: $1145,1969$.

31 Beshedr Robert M,D., Hendley Owen, Charlottesville V.: Severe pulmonary involvement in visceral Jarva migrans. Am. J. Dis. Child 12S: 599, 1973.

32 Lukens J.N.: Eosinophilia in children. Pediatr. Clin. NA 19: 969, 1972. 